\title{
Statistical Mechanics of the Chinese Restaurant Process: lack of self-averaging, anomalous finite-size effects and condensation
}

\author{
Bruno Bassetti ${ }^{1}$, Mina Zarei ${ }^{2}$, Marco Cosentino Lagomarsino ${ }^{1}$ and Ginestra Bianconi ${ }^{3}$ \\ 1 Universitá degli Studi di Milano, Dip di Fisica, via Celoria 16,20133 Milano, Italy \\ ${ }^{2}$ Department of Physics, Isfahan University of Technology, Isfahan 84156-83111, Iran \\ 3 Physics Department, Northeastern University, Boston 02115, MA ,USA
}

\begin{abstract}
The Pitman-Yor, or Chinese Restaurant Process, is a stochastic process that generates distributions following a power-law with exponents lower than two, as found in a numerous physical, biological, technological and social systems. We discuss its rich behavior with the tools and viewpoint of statistical mechanics. We show that this process invariably gives rise to a condensation, i.e. a distribution dominated by a finite number of classes. We also evaluate thoroughly the finite-size effects, finding that the lack of stationary state and self-averaging of the process creates realizationdependent cutoffs and behavior of the distributions with no equivalent in other statistical mechanical models.
\end{abstract}

PACS numbers: 89.75.Hc, 89.75.Da, 89.75.Fb

Despite of their extreme behavior, power-law tailed probability distributions empirically describe the partitioning (i.e. the organization into sub-classes) of a large variety of physical, biological, technological and social systems [1], and the degree distributions of many complex networks [2, 3]. The most extreme case are the systems where the power laws $p_{k}$ have exponent $\gamma$ between one and two. Indeed, if $\gamma \in[1,2]$ both the first and the second moment of the distribution diverge, meaning that the system is so biased that neither averages nor fluctuations are well-behaved. This seems to happen for example for words in a text or population of cities (Zipf's law $[1,4,5])$, the size of classes of homolog proteins, the out-degree of transcription networks [6], the frequency of family names [1], and of degree distribution of different social/technological networks [7].

While the processes leading to power-law distributions are diverse [1], there are only few available models that capture this behavior. In particular, it is useful to formulate models that help the understanding of these distributions in the framework of non-equilibrium growth laws. The paradigm is the Yule/Simon process [8, 9], which describes an evolving system of a growing number of elements, where the number of classes grows linearly with the elements. It is based on the general mechanism of the "Matthew effect", or "cumulative advantage": with time, more populated classes acquire new elements with higher relative rate. The Yule/Simon process generates powerlaw distributions with exponents $\gamma \in(2,3]$ [1]. Barabási and Albert [10] have shown that a similar mechanism can be used to generate power-law networks with the same exponents that grow and evolve through preferential attachment [2, 3, 10].

While in some systems where exponents $\gamma \leq 2$ are observed it can be argued that preferential attachment is present, this case is not predicted by the Yule/Simon/Barabási-Albert (YSBA) model. These distributions are more biased towards highly populated classes, so that in order to obtain this behavior one has to reweigh the balance of growth and preferential attachment in favor of the latter, and in particular consider processes where the number of classes grows sub-linearly with the number of elements.

The process we consider here, called Pitman-Yor, or Chinese Restaurant Process (CRP) [11, 12], has exactly this property, which makes it reproduce power-laws with $\gamma \in(1,2]$. It is commonly used in the mathematics literature, but relatively disregarded by the physics community, and in particular unexplored using the tools of statistical mechanics. It is defined as a discrete-time stochastic process generating a partition of a number elements in classes, such that each element belongs to a given class. In probability, the CRP is used for example as a prior in nonparametric Bayesian methods and its has been applied to multiple problems ranging from modeling texts to genetics and functional genomics 13, 14, 15, 16, 17]. It also maps to a non self-averaging stick-breaking process [12, 18]. Recently, we observed that CRP-like processes model well the evolution protein domain families [19] and reproduce the scaling laws found by genomics methods, which adds a strong motivation to explore them.

The mathematical characterization of the CRP has been carried out [12] with special attention to the asymptotic of the process at large times $T \rightarrow \infty$. In this Letter, we characterize this process with the tools of statistical mechanics. First, we argue that the CRP always exhibits a condensation phenomenon [20, 21, 22, 23, 24, 25, 26] with few classes dominating the total population. We relate the condensation observed in the CRP process to other known mechanisms taking place in the well-studied phenomenology of the Zero-Range Process [22], or in network models [21]. Second, we present a calculation that shows how the process behaves for large but finite times $T$, finding anomalous finite-size corrections to the asymptotic formulas. Unlike the YSBA model [27], the lack of 
self-averaging of the CRP determines for certain parameter values a nontrivial and realization-dependent finitesize behavior which is our main finding. Thus, the CRP fills two important gaps in the fundamental statistical mechanical understanding of non-self averaging phenomena, power-law distributions, and condensed states.

General Considerations. At each time $T$, the CRP generates a partition over integers $\{1,2, \ldots T\}$ into different classes. Differently from other models [21, 22], the number of classes $N(T)$ is a stochastic variable depending on the realization.

The process is anecdotally a problem of customers entering a Chinese Restaurant with table-sharing, where the number of tables and guests per table are unbounded. Assuming that in the restaurant there are $T$ customers sitting at $N(T)$ tables with $k_{i}$ customers in each table $i=1,2, \ldots N(T)$. At time $T$ a new customer enters the restaurant and either sits at table $i=1, \ldots, N(T)$ with probability $p_{i}$ (in this case $N(T+1)=N(T)$ ), or chooses a new table with probability $p_{N(T)+1}$ (in this case $N(T+1)=N(T)+1)$. The probability $p_{i}$ and $p_{N(T)+1}$ in the CRP are given by

$$
p_{i}=\frac{k_{i}-\alpha}{T+\theta} \quad p_{N(T)+1}=\frac{\alpha N(T)+\theta}{T+\theta},
$$

where $\theta>0$ and $\alpha \in[0,1)$.

As in the YSBA model, the CRP includes growth of elements $T$ and classes $N$, and a preferential attachment principle, because more populated tables are more likely to acquire new customers. However, in the CRP, growth of classes is not constrained to preferential attachment of new class members, but these two processes are decoupled, as witnessed by the fact that the probability to add a new table is not constant. This probability decays with the number of guests $T$, increasing the weight of "hub classes", which is the essential ingredient to reproduce power-law distributions with exponent lower than two. While models producing power-laws with $\gamma \in(1,2]$ exist [28], usually they lack the flexibility of the CRP in modulating this weight.

Rephrasing the YSBA model in terms of customers in a restaurant, the probabilities $p_{i}^{S}$ to sit at a non-empty table $i$ at time $T$ and the probability $p_{N(T)+1}^{S}$ to sit at a new table at time $T$ is $p_{i}^{S}=(1-\varepsilon) k_{i} / T, p_{N(T)+1}^{S}=\varepsilon$ where $k_{i}$ is the occupation number of table $i=1, \ldots, N(T)$. Note that this means that new tables are added with statistically independent moves, while in the CRP the addition of a new table is statistically dependent on the configuration of the partition [19].

The CRP has been studied extensively in the mathematical literature [12]. The occupation distribution in the limit $T \rightarrow \infty$ and $k$ finite and fixed is $F_{\infty}(k)=$ $\frac{\Gamma(k-\alpha)}{\Gamma(k+1) \Gamma(1-\alpha)}$. Furthermore, the statistics of the number of tables $N(T)$ has been characterized. The average value of the number of tables $\langle N(T)\rangle$ at time $T$ is given by 12 .

$$
\langle N(T)\rangle \simeq\left\{\begin{array}{lll}
\frac{\Gamma(\theta+1)}{\alpha \Gamma(\theta+\alpha)} T^{\alpha} & \text { for } & \alpha>0 \\
\theta \log (T+\theta) & \text { for } & \alpha=0
\end{array}\right.
$$

In the limit of large $T$, the full probability distribution for $N(T) \mathcal{P}(N(T))$ is known [12], when $\alpha=0$, to be a Gaussian of mean $m$ and standard deviation $\sigma^{2}$ with $m=\sigma^{2}=\theta \log (T)$. In the case $\alpha>0$, instead, the variable $s=N(T) / T^{\alpha}$ asymptotically in time follow the Mittag-Leffler distribution $g_{\alpha, \theta}(s)$ [12]. This point is particularly interesting [12, 29] because in the asymptotic limit, the Mittag-Leffler distribution has finite fluctuations, implying that the number of tables in the PitmanYor process with $\alpha>0$ is a non self-averaging quantity.

The CRP is always in a condensed state. We now provide an argument comparing the phenomenology of the CRP to models exhibiting condensation phenomena. Extending the validity of the asymptotic formula $F_{\infty}(k)$ for all values of $k$, we can estimate the occupation of the maximally occupied table in the CRP. We observe that this table has always occupation $k_{\max }=\mathcal{O}(T)$. In fact, since $F_{\infty}(k) \sim k^{-\alpha-1}$, we can evaluate the occupation $k_{\max }$ of the maximally occupied table by imposing the defining condition that that the fraction of tables with $k>k_{\max }$ must be of the order of $1 / N$, i.e.

$$
\sum_{k>k_{\max }} F_{\infty}(k) \simeq \frac{1}{N}
$$

Since in the Chinese restaurant process $N=\mathcal{O}\left(T^{\alpha}\right)$ if $\alpha>0$, and $N=\mathcal{O}(\log (T))$ if $\alpha=0$, in both cases this estimate indicates that the maximally occupied table has a finite occupation $k_{\max }=\mathcal{O}(T)$.

When the maximal occupation of a class is of the same order of the total number of elements in the partition, one says that the distribution is in a "condensed" phase. Reference models studied in the statistical physics community are the Zero-Range-Process (ZRP) and the BoseEinstein condensation of networks (BECN) 21]. In the BECN the condensation occurs on a single special node, for power-law degree distributions with exponent $\gamma=2$ as a consequence of the heterogeneity of the classes. In the ZRP, particles hop on 1-D lattice sites according to prescribed laws [22], generating partitions of elements into classes, i.e. clusters of particles, with power-law behavior and exponent $\gamma$. Depending on the dynamics and particle density, a condensation can occur in the ZRP, where one class becomes occupied by a finite fraction of elements.

It is instructive to illustrate the main differences between the condensation phenomena occurring in the ZRP and in the CRP: First, in the ZRP the exponent $\gamma$ of the distribution can be larger or smaller than 2, but the condensation occurs only if $\gamma>2$, while in the CRP a condensation always occurs and the distribution of the 
partition decays with an exponent $\gamma=1+\alpha<2$. Second, in the $\gamma>2 \mathrm{ZRP}$, the condensation transition is driven by the density of particles $\rho$ : If $\rho>\rho^{\star}$ there is a condensation, if $\rho<\rho^{\star}$ there is no condensation, and the condensate appears in order to balance between the imposed finite value of $\rho$ and the natural average value of the power-law. Conversely, in the CRP the mean density of elements always diverges, which, in the large $T$ limit imposes the existence of classes with a finite fraction of the total number of elements. Thus, a relevant difference between the ZRP (and BECN) and the CRP is that in the CRP there is a degenerate distribution but no phase transition. This situation closely resembles the so called "pseudo-condensation" found [30] where the condensation is characterized in a ZRP with non-extensive number of classes. However while in that case the scaling of the number of classes with the number of elements is chosen ad hoc, in the CRP this scaling is a natural outcome the process.

Conditioned Path Integral of the CRP and anomalous finite-size effects. At finite-sizes the $\alpha>0 \mathrm{CRP}$ shows an intriguing phenomenology, where the trend of individual realizations determines their distribution. This is visible from the finite-size scaling of the distribution $F(k, T)$. We thus study $F(k, N, T)$, with the additional condition of fixed number of tables $N$.

The probability of a partition of $T$ elements is the probability distribution at $T-1$ times the probability of an event at time $T$. Therefore the probability $P\left(\left\{k_{i}\right\}\right)$ of a process from time $T=1$ to time $T$, giving rise to an occupation of $N$ tables $i=\{1,2, \ldots N\}$, each one occupied by $k_{i}$ individuals, is given by the product of the probabilities (11) for each subsequent event. In particular this probability can be written as

$$
P\left(\left\{k_{i}\right\}\right)=C_{N, T}\left(\prod_{i} \frac{\Gamma\left(k_{i}-\alpha\right)}{\Gamma(1-\alpha)}\right) \delta_{T, \sum_{i} k_{i}},
$$

where $\delta$ is the Kronecker delta fixing the total number of customers and the constant $C_{N, T}$ is given by $C_{N, T}=\alpha^{N} \Gamma(N+\theta / \alpha) \Gamma(\theta) /[\Gamma(\theta / \alpha) \Gamma(T+\theta)]$. Most notably, the probability $P\left(\left\{k_{i}\right\}\right)$ of a process giving rise to the occupation numbers $\left\{k_{i}\right\}$, Eq. (3) is independent on the history of the process. In this case $P\left(\left\{k_{i}\right\}\right)$ is called a distribution of exchangeable random variables [12]. Moreover, since $P\left(\left\{k_{i}\right\}\right)$ takes a factorizable form, this probability distribution is also referred to as a Gibbs measure [12].

We can construct a conditioned path integral of this process by summing over all the histories keeping $T$ and $N$ constant. Since the events in the CRP are exchangeable [12], i.e. the probability is invariant for any permutation of the set of class indexes, we can sum over the histories in which the partition $\left\{k_{i}\right\}$ is generated in random order. To account for the number of these histories we introduce the multinomial prefactor $T ! /\left(\prod_{i} k_{i} ! N !\right)$.
This leads to the following expression for the partition function $Z_{N, T}$,

$$
Z_{N, T}=\frac{1}{N !} \sum_{\left\{k_{i}\right\}_{i=1, \ldots N}} \frac{T !}{\prod_{i} k_{i} !} P\left(\left\{k_{i}\right\}\right) .
$$

Similarly, the probability $F(k, N, T)$ that in a process studied at time $T$ when $N$ tables are full, a random table is occupied by $k$ guests reads

$F(k, N, T)=\frac{1}{Z_{N, T}} \sum_{\left\{k_{i}\right\}_{i=1, \ldots N}} \frac{T !}{N ! \prod_{i} k_{i} !} \delta_{k_{1}, k} P\left(\left\{k_{i}\right\}\right)$.

Or equivalently,

$$
F(k, N, T)=\frac{Z_{N, T-k}}{Z_{N, T}} \frac{\Gamma(k-\alpha)}{\Gamma(k+1) \Gamma(1-\alpha)}
$$

Roughly, the ratio appearing at the r.h.s. in this equation is related to the power-law behavior, while the rest gives the finite-size corrections. The function $Z_{N, T}$ can be evaluated, for large $T$, with a saddle-point approximation of the integral

$Z_{N, T}=\int \frac{d \omega}{2 \pi} e^{i \omega T}\left(\sum_{k=1}^{T} \frac{\Gamma(k-\alpha)}{\Gamma(1-\alpha) \Gamma(k+1)} e^{-i \omega k}\right)^{N}$,

where the integration over $\omega$ comes from the Fourier representation of the Kronecker delta of Eq. 3, and the saddle point $i \omega^{\star}=\hat{\omega}:=\chi / T$ satisfies the equation

$$
\frac{T}{N}=\frac{H_{1}(T, \alpha, \chi / T)}{H_{0}(T, \alpha, \chi / T)}
$$

where

$$
H_{n}(T, \alpha, \omega)=\sum_{m=1}^{T} m^{n} \frac{\Gamma(m-\alpha)}{\Gamma(1-\alpha) \Gamma(m+1)} e^{-\omega m} .
$$

For $\alpha>0$, the solution to the saddle-point equation (8), $\chi=\chi(N, T, \alpha)$ in the limit $T \rightarrow \infty$ depends on $N, T$ only through the realization-specific variable $s=N / T^{\alpha}$. In order to show this, we first observe that the scaling of $H_{n}(T, \alpha, \chi / T)$ with $T$, can be studied by approximating integral to sums in their definition. Secondly, we show that the functions $H_{n}(T, \alpha, \chi / T)$ can be expressed as

$$
\begin{aligned}
& H_{n}(T, \alpha, \chi / T) \simeq \frac{1}{\Gamma(1-\alpha)} \frac{\left[T^{n-\alpha}-1\right]}{(n-\alpha)} \\
& +\frac{1}{\Gamma(1-\alpha)} T^{n-\alpha} \int_{0}^{1} d t \frac{1}{t^{\alpha+1-n}}\left(e^{-\chi t}-1\right),
\end{aligned}
$$

where we have added and subtracted a term of the type $H_{n}(T, \alpha, 0)$. Consequently, for large $T, H_{1}(T, \alpha, \chi / T) \rightarrow$ $T^{1-\alpha} h_{1}(\alpha, \chi)$ while $H_{0}(T, \alpha, \chi / T) \rightarrow h_{0}(\alpha)$. Inserting these relations in the saddle point-equation (8), and taking $N=s T^{\alpha}$ we obtain

$$
\frac{1}{s}=\frac{h_{1}(\alpha, \chi)}{h_{0}(\alpha)}
$$


proving that $\chi=\chi(s, \alpha)$ in the large $T$ limit for $\alpha>0$.

The final expression for $Z_{N, T}$ is therefore given by

$$
Z_{N, T} \simeq \frac{C_{N, T}}{N !} e^{\chi(s, \alpha)} \frac{e^{N \log \left(H_{0}(\alpha, \chi(s, \alpha) / T, T)\right)}}{\sqrt{N J(T, \alpha, \chi / T)}}
$$

where we evaluated the saddle point up to the second order, and the function $J(T, \alpha, \chi / T)$ is given by

$$
J(T, \alpha, \chi / T)=\left.\frac{\partial^{2} \log \left(H_{0}(T, \alpha, \omega)\right.}{\partial \omega^{2}}\right|_{\omega=\chi / T} .
$$

A similar procedure applies to the evaluation of $Z_{N, T-k}$, with $\chi^{\prime}=\omega^{\prime} /(T-k)$ satisfying the saddle point equation

$$
\frac{T-k}{N}=\frac{H_{1}\left(T-k, \alpha, \chi^{\prime} /(T-k)\right)}{H_{0}\left(T-k, \alpha, \chi^{\prime} /(T-k)\right)} .
$$

Following arguments similar to the one provided for the scaling of $\chi$, we can show that $\chi^{\prime}=\chi^{\prime}(k, N, T, \alpha)$ at the saddle point. Equation (14) depends on $k, N, T$ only through the variables $s=N / T^{\alpha}$ and $k / T$, i.e. $\chi^{\prime}=\chi^{\prime}(s, k / T, \alpha)$. Following a similar reasoning to he one we adopted for proving that the function $Z_{N, T}$ in Eq. (12) depends exclusively on the parameters $s, \alpha$, it is possible to show that the function $Z_{N, T-k}$ only depends on $s, k / T$, $\alpha$, i.e. $Z_{N, T-k}=\phi(s, k / T, \alpha)$.

Therefore, taking in (6) the large $k, T$ limit with $k / T=$ $\mathcal{O}(1), F(k, N, T)$ for $\alpha>0$ satisfies the scaling relation

$$
\begin{aligned}
T^{\alpha+1} F(k, N, T) & =T^{\alpha+1} \frac{Z_{N, T-k}}{Z_{N, T}} \frac{\Gamma(k-\alpha)}{\Gamma(k+1) \Gamma(1-\alpha)} \\
& =\left(\frac{T}{k}\right)^{1+\alpha} q\left(k / T, s=N / T^{\alpha}, \alpha\right)(15)
\end{aligned}
$$

where the function $q$ (containing $\chi^{\prime}, \chi$, and the second order corrections) represents the finite-size corrections to the asymptotic behavior. These findings shed light on the absence of self-averaging in the process. At any given time $T$ the process will have finite fluctuations, persistent also in the limit of $T \rightarrow \infty$. These fluctuations depend on the non stationarity of the process, and on the non self-averaging value of the number of classes $N$. Therefore the process, if conditioned on the number of classes $N$, shows fluctuations that go to zero as $T \rightarrow \infty$. Figure 1 compares simulations with the analytical predictions of Eq. (6). The figure shows that the finite-size correction to the power-law tail $\sim 1 / k^{1+\alpha}$, for some $s$ and large $k / T$ may increase its value, giving rise to an anomalous "bump" in the distribution. On the other hand, this local maximum never develops into a concentrated "condensate", and for $k / T \rightarrow 1$, for any $s$, the cutoff $q$ always dampens $F$.

In conclusion, we have presented a statistical mechanics study of the Chinese Restaurant Process, which generates power-law distributions with exponents $\gamma \in(1,2]$ by nonequilibrium growth, a condensation phenomena,

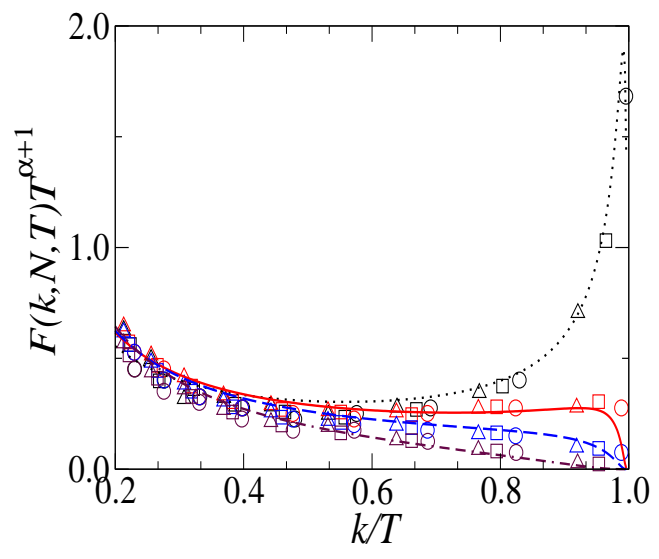

FIG. 1: (Color online) Rescaled distribution of the occupation numbers in the Chinese Restaurant Process with $\alpha=0.1$ and $\theta=1$ at different times $T$ and number of tables $N=$ $s T^{\alpha}$. We report the log-binned distribution $F(k, N, T)$ for $s=N / T^{\alpha}$ with $s=3.5$ (black symbols, dotted line) $s=5$ (red symbols, solid line) $s=7.5$ (blue symbols, dashed line) $s=10$ (brown symbols, dashed-dot line). The rescaled data are shown for processes with $T=2500$ (triangles), $T=5000$ (squares), $T=10^{4}$ (circles). The solid line show the analytical solutions calculated by solving the saddle point equations (8), (14) for $T=2500$.

absence of selfaverging, and anomalous finite-size effects. We believe that this rich process will be of importance for future developments of the field where these trends occur, i.e. biological evolution, complex systems, spinglasses and nonequilibrium phenomena.

G.B acknowledges support from the IST STREP GENNETEC contract number 034952.

[1] M. E. J. Newman, Contemporary Phys. 46, 323 (2005).

[2] R. Albert and A.-L. Barabási, Rev. Mod. Phys. 74, 47 (2002).

[3] S. N. Dorogovtsev and J.F. F. Mendes, Evolution of networks: From Biological Nets to the Internet and the $W W W$ (Oxford University Press, Oxford,2003).

[4] G. K. Zipf, Human Behavior and the Principle of Least Effort (Addison-Wesley Press, Inc. Cambridge, 1949).

[5] M. Marsili and Yi-Cheng Zhang, Phys. Rev. Lett.80, 2741 (1998).

[6] N. Guelzim, S. Bottani , P. Bourgine and F. Képés, Nature Genetics31,60 (2002).

[7] H. Seyed-allaei, G. Bianconi and M. Marsili, PRE 73, 046113 (2006).

[8] G. U. Yule, Philos. Trans. R. Soc. London B 213, 21 (1925).

[9] H. A. Simon, Biometrika, 42, 425 (1955).

[10] A.-L. Barabási and R. Albert, Science 286, 509 (1999).

[11] J. Pitman and M. Yor, Annals of Prob.25,855(1997)

[12] J. Pitman, Combinatorial Stochastic Processes (Lecture Notes in Mathematics Vol 1875, Springer-Verlag,Berlin, 
2006).

[13] R. M. Neal, Jour. Comp. Graph. Stat. 9, 249 (2000).

[14] D. M. Blei, T. L. Griffiths, M. I. Jordan and J. B. Tenenbaum, in Adv. Neural Inf. Process. Syst. 16, 17,2003.

[15] Z. S. Qin, Bioinformatics, 221988 (2006).

[16] E. P. Xing, M. I. Jordan and R. Sharan, Jour. Comput. Biol. 14267 (2007).

[17] W. J. Ewens, Theor. Popul. Biol.3 87, (1972).

[18] B. Derrida and H. Flyvbjerg, J. Phys. A :Math. Gen.20 5273 (1987).

[19] M. Cosentino Lagomarsino, A. L. Sellerio, P. D. Heijning, B. Bassetti, Genome Biology 10:R12 (2009).

[20] P. Bialas, Z. Burda D. Johnston, Nucl. Phys. B 493,505 (1997).

[21] G. Bianconi A.-L. Barabasi, Phys. Rev. Lett. 86,5632 (2001).

[22] M.R. Evans T. Hanney, J. Phys. A 38 (2005).
[23] J. D. Noh, PRE 72, 056123 (2005).

[24] S.N. Majumdar, M.R. Evans, R. K. P. Zia, Phys. Rev. Lett.94 180601 (2005).

[25] S.N. Dorogovtsev, Rev. Mod. Phys. 801275 (2008).

[26] C. Godréche and J. M. Luck, J. Phys. A: Math Gen 38,7215 (2005).

[27] P. L. Krapivsky S. Redner, J. Phys. A 35, 9517 (2002).

[28] A.E. Scheidegger, Bull. Int. Acco. Sci. Hydrolo., 12, 15 (1967); H. Takayasu, Phis Rev Lett, 63, 2563 (1989); D. Dhar and R. Ramaswamy, Phis Rev Lett, 63, 1659 (1989); S.N. Majumdar and C. Sire, Phis Rev Lett, 71, 3732 (1993).

[29] H. Yamato and M. Sibuya Bull.Inform. Cybernetics, 32, 1 (2000).

[30] M. Evans et.al., J. Stat. Phys. 123, 357 (2006). 